\title{
Kidney epithelium specific deletion of kelch-like ECH-associated protein 1 (Keap1) causes hydronephrosis in mice
}

\author{
Sanjeev Noel', Lois J. Arend², Samatha Bandapalle', Sekhar P. Reddy ${ }^{3}$ and Hamid Rabb¹,4*
}

\begin{abstract}
Background: Transcription factor Nrf2 protects from experimental acute kidney injury (AKI) and is promising to limit progression in human chronic kidney disease (CKD) by upregulating multiple antioxidant genes. We recently demonstrated that deletion of Keap 1, the endogenous inhibitor of Nrf2, in T lymphocytes significantly protects from AKI. In this study, we investigated the effect of Keap1 deletion on Nrf2 mediated antioxidant response in the renal tubular epithelial cells.
\end{abstract}

Methods: We deleted Keap 1 exon 2 and 3 in the renal tubular epithelial cells by crossing Ksp-Cre mice with Keap 1 floxed $\left(K_{\left.e a p 1^{\mathrm{f} / f}\right)}\right.$ mice. Deletion of Keap1 gene in the kidney epithelial cells of Ksp-Keap ${ }^{1-1}$ mice and its effect on Nrf2 target gene expression was performed using PCR and real-time PCR respectively. Histological evaluation was performed on H\&E stained sections. Complete blood count, serum and urine analysis were performed to assess systemic effects of defective kidney development. Student's T test was used to determine statistical difference between the groups.

Results: Ksp-Cre resulted in the deletion of Keap1 exon 2 and 3 and subsequent upregulation of Nrf2 target genes, $\mathrm{Nqo1}$, Gclm and Gclc in the kidney epithelial cells of Ksp-Keap 1-- mice at baseline. Renal epithelial cell specific deletion of Keap 1 in $\mathrm{Ksp}_{\mathrm{s}} \mathrm{Keap}^{-1-}$ mice caused marked renal pelvic expansion and significant compression of medullary parenchyma consistent with hydronephrosis in both (3 month-old) males and females. Kidneys from 6 month-old Ksp-Keap 1/- mice showed progressive hydronephrosis. Hematological, biochemical and urinary analysis showed significantly higher red blood cell count $(p=0.04)$, hemoglobin $(p=0.01)$, hematocrit $(p=0.02)$, mean cell volume $(p=0.02)$ and mean cell hemoglobin concentration $(p=0.003)$ in $K s p$-Keap $1^{-1 /}$ mice in comparison to Keap $1^{f / f}$ mice.

Conclusions: These unexpected findings demonstrate that Keap1 deletion in renal tubular epithelial cells results in an abnormal kidney development consistent with hydronephrosis and reveals a novel Keap1 mediated signaling pathway in renal development.

Keywords: Keap1-Nrf2 pathway, Hydronephrosis, Kidney epithelial cells, Kidney development

\section{Background}

The Keap1-Nrf2 cytoprotective response is critical to combat reactive oxygen species (ROS) and electrophiles generated during endogenous and exogenous stresses [1-3]. Keap1 (Kelch-like ECH-associated protein 1) is a repressor protein that regulates transcriptional activity of Nrf2 (nuclear factor erythroid 2-related factor 2) by

\footnotetext{
* Correspondence: hrabb1@jhmi.edu

'Department of Medicine, Johns Hopkins University, Baltimore, MD, USA

${ }^{4}$ Division of Nephrology, Department of Medicine, Johns Hopkins University,

Ross 965720 Rutland Avenue, Baltimore, MD 21205, USA

Full list of author information is available at the end of the article
}

retaining it in the cytoplasm and maintaining its homeostatic level by directing it to proteasomal degradation [4-6]. However, during stress conditions, such as ischemic and nephrotoxic injury, Nrf2 is released in to the nucleus to up regulate the transcription of cytoprotective genes. An insufficient Nrf2 activity has been shown to worsen ischemia induced kidney injury and accelerate disease progression largely due to an attenuated antioxidant response [1, 7]. Nrf2 levels have also been shown to decrease with ageing and correlate with the progression of many human diseases [8]. 
Table 1 Primer information for PCR based confirmation of Cre, Keap 1 floxed and keap1 deleted allele status

\begin{tabular}{llll}
\hline S No. & Primer name & Sequence 5'-3' & Product size \\
\hline 1 & Generic Cre Forward Primer & GCGGTCTGGCAGTAAAAACTATC & 100bp \\
& Generic Cre Reverse Primer & GTGAAACAGCATTGCTGTCACTT & keap1 floxed allele: 383bp \\
& Keap 1flox Forward Primer & CGAGGAAGCGTTGCTTAC & WT allele: 2954bp \\
3 & Keap 1flox Reverse Primer & GAGTCACCGTAAGCCTGGTC & Truncated allele: 288bp \\
& Keap1 deletion Forward Primer & GAGTCCACAGTGTGTGGCC & \\
& Keap1 deletion Reverse Primer & GAGTCACCGTAAGCCTGGTC &
\end{tabular}

Attempts to up regulate global Nrf2 levels have been difficult because homozygous knock out of Keap1 gene is lethal. Whole body Keap1 $1^{-/-}$mice do not survive beyond 21 days postnatal due to progressive asthenia as a result of hyperkeratosis of esophagus and forestomach [9]. However, recent use of Cre-LoxP technology has facilitated researchers to up regulate Nrf2 activity in a tissue specific manner [10]. We recently generated mice with increased Nrf2 activity in T lymphocyte by genetically deleting Keap1 and found these mice to be significantly protected from ischemia reperfusion induced AKI [11].

In the present study we deleted Keap1 in renal epithelial cells, specifically in the distal convoluted tubules and collecting ducts, primarily to accomplish kidney epithelial cell specific up regulation of Nrf2 mediated antioxidant response and to study its effect on ischemic kidney injury. Surprisingly, renal epithelial cell specific deletion of Keap1 resulted in significant developmental defects in the collecting system. These anatomical defects were also accompanied by polycythemia. In summary, these data demonstrate that Keap1 may be involved in normal kidney development and that a defective Keap1 results in hydronephrosis.

\section{Methods}

Generation and characterization of Ksp-Keap $1^{-/-}$mice Kidney epithelial cell specific Keap1-deficient (henceforth referred to as $K s p$-Keap $1^{-/-}$) mice were generated by breeding $\operatorname{Keap1}^{\mathrm{f} / \mathrm{f}}$ mice with Ksp-Cre mice. Keap1 ${ }^{\mathrm{f} / \mathrm{f}}$ mice used for these studies have already been characterized [10, 12]. Male Ksp-Cre mice were purchased from Jackson Laboratories and a detail description about its generation is provided on their website (http://jaxmice.jax.org/strain/ 012237.html). The Cre transgene in Ksp-Cre mice is under the control of cadherin 16 (Cdh16 or Ksp-cadherin)
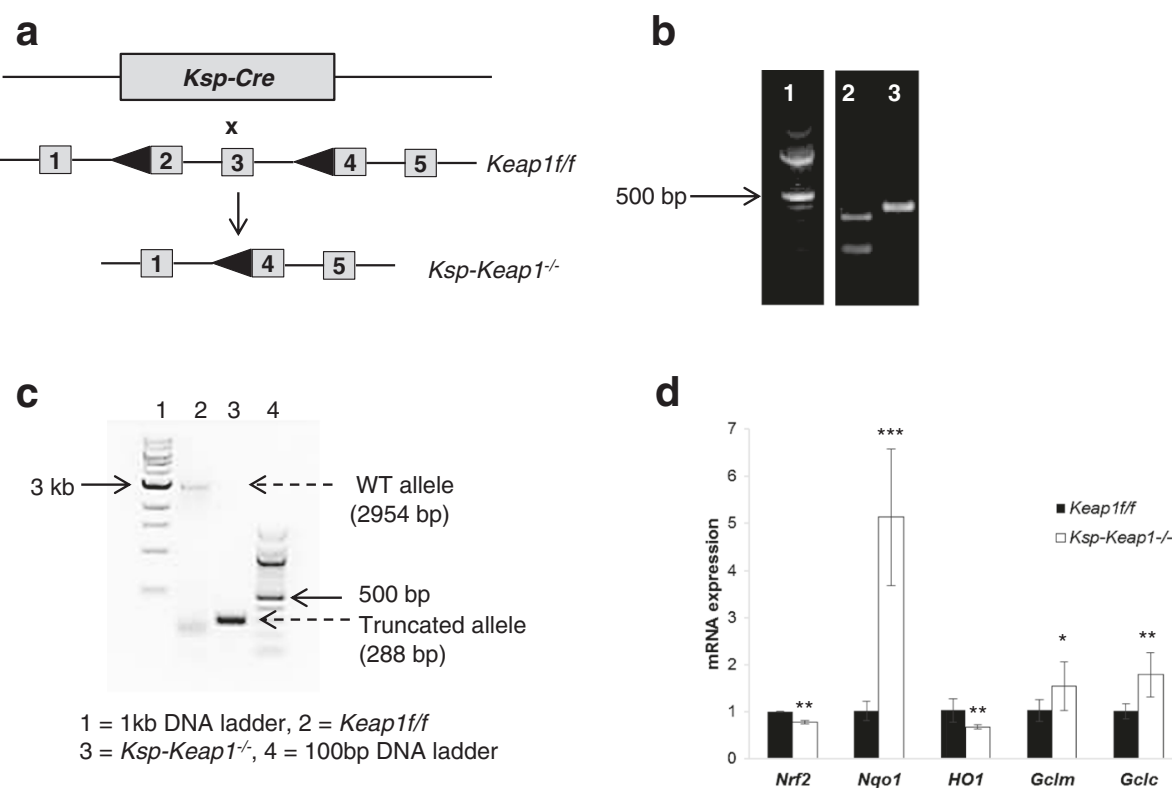

Fig. 1 Generation and characterization of Ksp-Keap $1^{-1-}$ mice. a Ksp-Cre mice were crossed with Keap $1^{f / f}$ mice to generate Ksp-Keap $1^{-1}$ mice. b Mice were genotyped to confirm the presence of Cre and floxed Keap1 allele using Cre and flox specific primers. c Ksp-Cre mediated deletion of Keap 1 exon 2 and 3 resulted in a truncated allele (288 bp) in comparison to WT allele (2954 bp). d mRNA analysis of Nrf2 targets showed increased expression on Naol ( $p \leq 0.0001)$, Gclm ( $p \leq 0.05)$ and Gclc ( $p \leq 0.001$ ) but reduced expression of HO-1 ( $p=\leq 0.001)$ and Nrf2 ( $p=\leq 0.001)$. b Lane $1=100$ bp DNA ladder, lane $2=324$ bp internal positive control and $100 \mathrm{bp}$ Cre and lane $3=383$ bp Keap 1 floxed. $\mathbf{c}$ Lane $1=1 \mathrm{~kb}$ DNA ladder, lane $2=2954$ bp WT allele $($ Keap $1 f / f)$, lane $3=288$ bp truncated allele $\left(K_{\left.s p-K e a p 1^{1-}\right)}\right)$ and lane $4=100$ bp DNA ladder 
promoter and specifically deletes any LoxP flanked gene in the renal epithelium. Mice were genotyped to confirm the presence of Cre transgene, flox status using PCR primer sets listed in Table 1.

\section{Isolation of kidney epithelial cells}

Kidney epithelial cells were isolated using a previously described method (2) to ascertain deletion of Keap1 and to quantify its effect on Nrf2 activity. Briefly, kidneys were isolated, from anesthetized Keaplf/f $(n=3)$ and Ksp-Keap1 ${ }^{-/}$ mice $(n=3)$ following exsanguination, minced and incubated in dispase II for $45 \mathrm{~min}$ at $37{ }^{\circ} \mathrm{C}$. Minced tissue was passed through $100 \mu \mathrm{m}$ cell strainer followed by $70 \mu \mathrm{m}$ strainer, resuspended in DMEM/HEPES and incubated for 30 min on IgG coated plates at $37{ }^{\circ} \mathrm{C}$ in $\mathrm{CO}_{2}$ incubator to remove macrophages and other leukocytes. Non-adherent cells were further incubated on $100 \mathrm{~mm}$ petri dishes for $2 \mathrm{~h}$ to remove fibroblasts and remaining nonadherent cells were used for DNA and RNA isolation.

\section{Keap1 deletion PCR}

To confirm Ksp-Cre mediated deletion of Keap1 exon 2 and 3, DNA was isolated from kidney epithelial cells using DNA isolation kit (QIAGEN). Keap1 deletion specific primers (Table 1) spanning exon 2 and 3 were used to detect intact or truncated Keap1 alleles using PCR.

\section{Nrf2 target gene expression analysis}

RNA was isolated from kidney epithelial cells using RNA mini kit (QIAGEN) to quantify Nrf2 and its target gene expression at mRNA level. We measured Nrf2, Nqo1, HO-1, Gclm and Gclc levels with realtime PCR using gene specific Taqman primer and probe sets (Life Technologies). Actin was used to normalize gene expression data and fold change was calculated by delta delta CT method as described previously (11).

\section{Kidney histology}

Upon sacrifice the kidneys were harvested and fixed with $10 \%$ buffered formalin phosphate and embedded with paraffin for histological evaluation. Tissue sections $(5 \mu \mathrm{m})$ were stained with hematoxylin and eosin (H\&E) and examined for gross histological abnormalities by an expert renal pathologist (LJA) blinded to the groups.
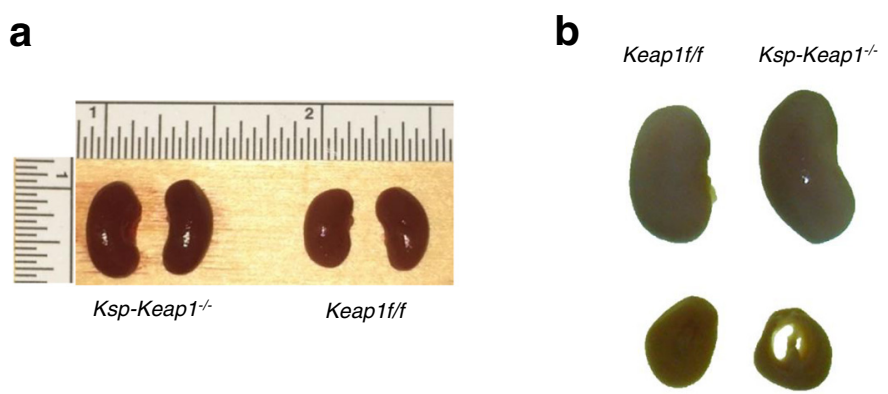

C
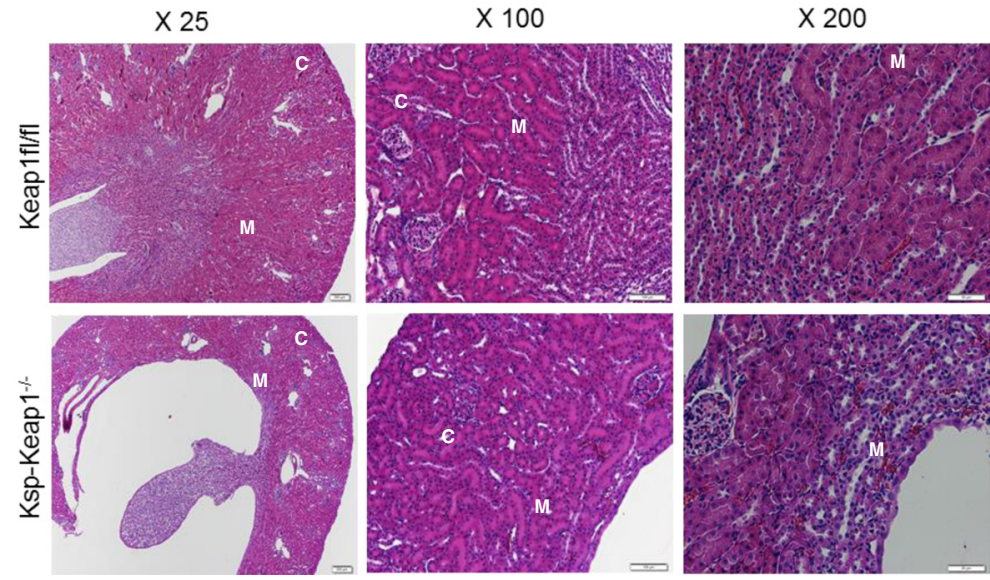

Fig. 2 Anatomical and histological studies of 3 month old Ksp-Keap $1^{-1-}$ and Keap $1^{f / f}$ mice. a Kidneys from Ksp-Keap $1^{-1-}$ mice were slightly bigger. b A transverse section through kidney show missing or compressed medullary tissue in Ksp-Keap ${ }^{-1-}$ kidney. c Hematoxylin and eosin (H\&E) stained kidney section at different levels of magnification showing normal cortex, however significant medullary tissue is missing from $\mathrm{Ksp}^{-K e a p} \mathrm{r}^{-1}$ kidney. The size of the bars is $200 \mu \mathrm{m}, 100 \mu \mathrm{m}$ and $50 \mu \mathrm{m}$ for X25, X100 and X200 images respectively 


\section{Complete blood, serum and urine analysis}

Blood was collected in microtainers with or without $\mathrm{K}_{2}$ EDTA (BD). Urine samples were collected by placing the mice on a microtitre plate for $60 \mathrm{~min}$. Uncoagulated blood samples were analyzed with HemaVet multispecies hematology instrument (Drew Scientific) to measure percentage of leucocytes, platelets, erythrocytes hemoglobin, mean cell volume and mean cell hemoglobin. Biochemical assessment of serum chloride and urinary calcium and total protein was done in automated VetAce clinical chemistry system (Alfa Wasserman Diagnostic Technologies).

\section{Data analysis}

Means were compared by a paired, two-tailed student's $t$ test for a single comparison between two groups. Statistical significance was accepted at a $p$ value $\leq 0.05$.

\section{Results and discussion}

PCR based characterization confirmed the deletion of Keap 1 exon 2 and 3 in Ksp-Keap1 ${ }^{-/}$mice (Fig. 1b, c). Furthermore, Nrf2 target gene Nqo1, Gclm and Gclc were significantly upregulated in kidney epithelial cell from Ksp-Keap $1^{-/-}$mice compared to Keaplf/f mice (Fig. 1d), which is consistent with our previous findings in T lymphocytes (11). Interestingly, mRNA levels of Nrf2 and HO- 1 were found to be reduced in the kidney epithelial

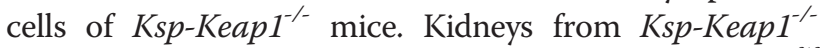
mice were slightly larger than the age matched $K e a p 1^{\mathrm{f} / \mathrm{f}}$ control mice and showed unexpected gross developmental defects (Fig. 2a, b). Furthermore, transverse sections of the kidneys of all $\mathrm{Ksp}$-Keap ${ }^{-/-}$mice $(n=5)$ revealed moderate to marked renal pelvic expansion and significant compression of medullary parenchyma in comparison to Keap $1^{\mathrm{f} / \mathrm{f}}$ kidneys $(n=5)$. Histological investigation of $\mathrm{Ksp}_{\mathrm{K}} \mathrm{Keapr}^{-1}$ kidneys with H\&E stained sections revealed largely missing or underdeveloped medullary region whereas the cortical region appeared to be normal (Fig. 2c). We did not see any obstruction in the ureters of these mice, which is the most common cause of hydronephrosis in humans. All the other organs (liver, spleen, heart, skin, brain etc.) were grossly normal and none of them showed any signs of histological abnormality indicating
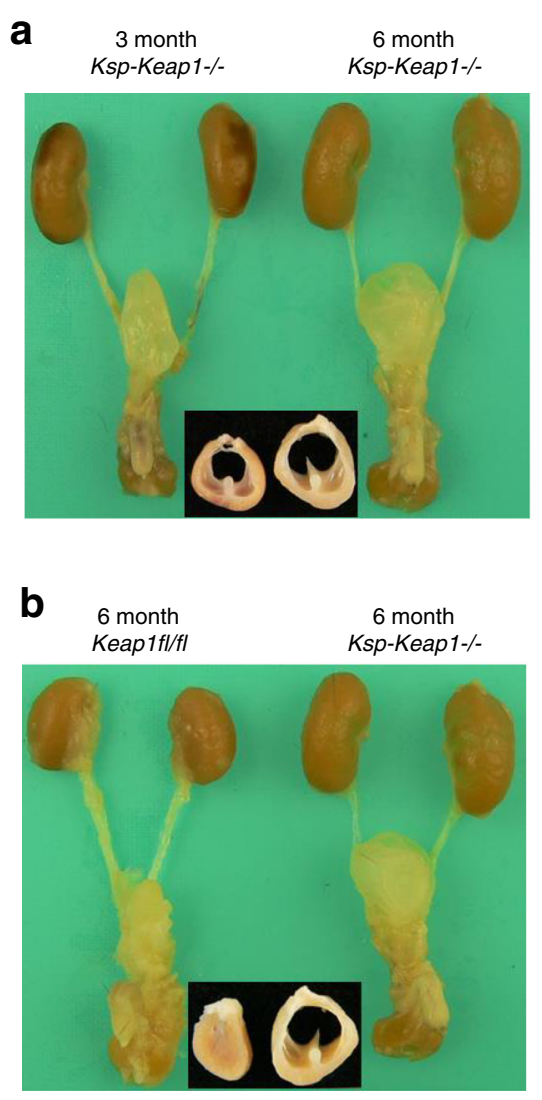
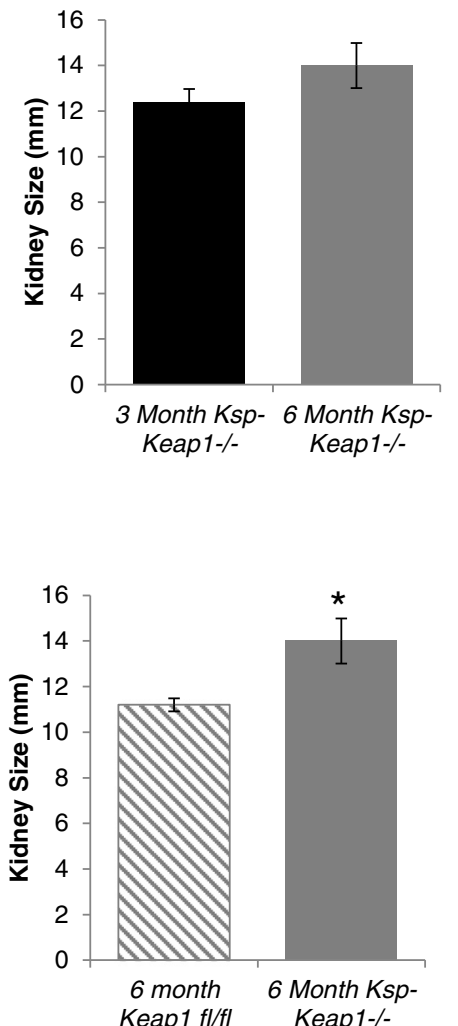

Fig. 3 Comparison of kidney size in 6 month old Ksp-Keap $1^{-1-}$ mice $(n=5)$ to 3 month old Ksp-Keap $1^{-/-}(n=4)$ and 6 month old Keap $1^{f / f}$ mice $(n=5)$. Kidneys of 6 month old Ksp-Keap $1^{-1}$ mice were significantly bigger than age matched Keap $1^{f / f}$ mice $(14 \pm 0.8 \mathrm{vs} 11.2 \pm 0.3 \mathrm{~mm}, p=0.05)$. Although Kidneys of 3 month old Ksp-Keap ${ }^{-1-}$ mice were smaller than 6 month old Ksp-Keap $1^{-1-}$ mice the difference was not statistically significant $(12.4 \pm 0.6$ vs $14 \pm 0.8, n s)$. Panels $\mathbf{a}$ and $\mathbf{b}$ showing transverse sections of kidneys with hydronephrosis from 3 month and 6 month old mice. Data are presented as mean \pm standard deviation (SD) 
that Ksp-cre specifically targets kidney epithelial cells. Furthermore, the kidneys from 6 month-old Ksp-Keap1 ${ }^{-/}$ mice $(n=5)$ showed progressive hydronephrosis in comparison to 3 month-old Ksp-Keap $1^{-/}$mice $(n=4)$ (Fig. 3a) and were significantly bigger $(p=0.05)$ than kidneys from 6 month-old Keap1 ${ }^{\mathrm{f} / \mathrm{f}}$ control mice (Fig. 3b). We observed similar findings in 3 month and 6 month-old female mice, indicating that Keap1 deletion affects kidney development in both sexes.

Complete blood count (CBC) analysis of 3 week old male mice ( $n=3$ per group) did not reveal any difference in leukocyte and platelet populations (Fig. 4a, b) however, there was significantly higher red blood cells $(p=0.04)$, hemoglobin $(p=0.01)$, hematocrit $(p=0.02)$, mean cell volume $(p=0.02)$ and mean cell hemoglobin concentration $(p=0.003)$ in Ksp-Keap1 ${ }^{-1-}$ mice as compared to Keap $^{\mathrm{f} / \mathrm{f}}$ control mice of similar age (Fig. 4c). Furthermore serum chloride levels was significantly higher $(p=0.05)$ in $\mathrm{Ksp}_{\mathrm{Keap1}} \mathrm{K}^{-1-}$ mice as compared to Keap1 ${ }^{\mathrm{f} / \mathrm{f}}$ control mice (Fig. 4d). Additionally, urinary calcium $(p=0.02)$ and total protein $(p=0.007)$ were significantly lower in Ksp-Keap $1^{-1-}$ mice as compared to control mice (Fig. 4e). Our preliminary observation in older ( $\geq 6$ months) Ksp-Keap $1^{-/-}$mice indicate that Keap1 deletion results in progressive kidney damage that completely destroys normal kidney tissue.

In the present study, we generated mice with renal epithelial cell specific deletion of Keap1 by crossing Ksp-Cre mice with $\mathrm{Keap1}^{\mathrm{f} / \mathrm{f}}$ mice to primarily up regulate Nrf2 in kidney epithelial cells and to examine its effect on ischemic kidney injury. Cre recombinase in $\mathrm{Ksp}$-Cre mice is expected to delete any LoxP flanked gene in epithelial cells of developing nephrons, ureteric bud, mesonephric tubules, Wolffian duct, and Mullerian duct. In the adult

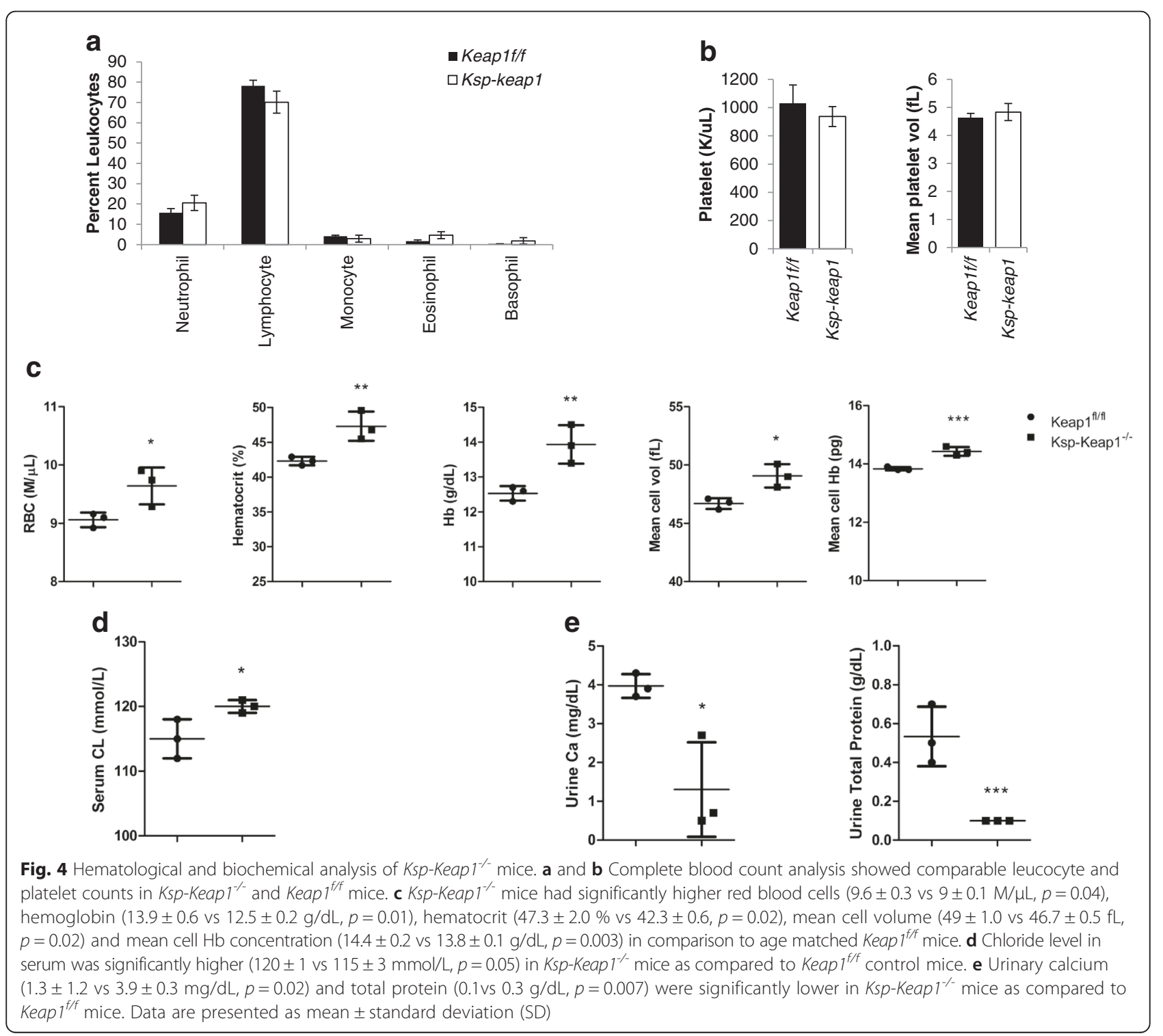


mouse Cre expression is limited to the renal tubules especially the collecting ducts, loops of Henle and distal tubules [13]. To our surprise, we observed marked renal pelvic expansion and significant compression of medullary parenchyma in kidneys from Ksp-Keap $1^{-/}$mice that was consistent with hydronephrosis. Furthermore, we found kidneys of both male and female mice were affected indicating that Keap 1 deletion is deleterious in both sexes.

It is unclear how Keap1 regulates or is involved in normal kidney development. Several other deficiencies such as Egf receptor, Claudin-4, Dlg1 and 17q12 microdeletion have also been linked to abnormal kidney development [14-17]. Moreover, in a previous human case report Stark et al. [18] presented similar findings in a 16 year old white male with chronic kidney disease and a history of obstructive uropathy. The patient had high red blood cells count and high erythropoietin but normal platelet and leukocyte count. There were no symptoms of cardiac, cerebral or pulmonary abnormalities. These human findings are very similar to our present finding.

Hydronephrosis results in significant tissue compression that is stretched out but not destroyed. This compression is thought to lead to local ischemia that stimulates erythropoietin production by cortical cell that subsequently result in increased erythropoiesis $[19,20]$. The elevated red blood cell count and hemoglobin is believed to be an effect of decreased oxygen delivery in the compressed hydronephrosed kidney tissue [21]. Events downstream of the oxygen-sensitive transcription factor are involved in the erythropoietin gene expression, including the production of specific transcription factor such as hypoxia-inducible factor 1 (HIF-1) [22]. A hypoxic stimulus increases the number of erythropoetin-producing cells in the cortex of kidney, but not the amount of erythropoietin produced per cell. These symptoms are corroborated by other finding indicating that the presence of hydrophephrosis, due to multiple etiologies decreases oxygen delivery with subsequent increase in erythropoietin production.

\section{Conclusions}

In conclusion, our unexpected finding may suggest a novel role for Keap1 mediated signaling pathway in renal development and indicate that absence of Keap1 in renal tubular epithelial cells significantly affects normal kidney development leading to hydronephrosis. Furthermore, the differences in $\mathrm{CBC}$ and other serum and urinary markers measured may indicate secondary systemic effects of hydronephrotic kidneys. Understanding the interaction between Keap1 and kidney development warrants further studies.

\section{Acknowledgments}

The authors are grateful for the generous research support of Mr. Rogelio Miro of Panama.
Funding

This work was funded and supported by the National Institutes of Health grant RO1DK084445

\section{Availability of data and materials}

We agree to provide resource or data related to this work at the expense of the requester.

\section{Authors' contributions}

SN, SPR and HR designed research; SN and SB performed experiments; LA performed histological assessment of kidney samples; SN, and HR analyzed data and wrote the paper. All authors read and approved the final manuscript.

\section{Competing interests}

The authors declare that they have no competing interests.

\section{Consent for publication}

Not applicable.

\section{Ethics approval and consent to participate}

All animal procedures were approved and conducted in compliance with Johns Hopkins animal ethics committee guidelines. Our manuscript reporting adheres to the ARRIVE guidelines in accordance with BioMed Central editorial policies.

\section{Author details}

'Department of Medicine, Johns Hopkins University, Baltimore, MD, USA. ${ }^{2}$ Department of Pathology, Johns Hopkins University, Baltimore, MD, USA. ${ }^{3}$ Department of Pediatrics, College of Medicine, University of Illinois, Chicago, IL, USA. ${ }^{4}$ Division of Nephrology, Department of Medicine, Johns Hopkins University, Ross 965720 Rutland Avenue, Baltimore, MD 21205, USA.

Received: 20 August 2015 Accepted: 19 July 2016

Published online: 02 August 2016

\section{References}

1. Liu M, Grigoryev DN, Crow MT, Haas M, Yamamoto M, Reddy SP, et al. Transcription factor Nrf2 is protective during ischemic and nephrotoxic acute kidney injury in mice. Kidney Int. 2009;76:277-85.

2. Liu M, Reddy NM, Higbee EM, Potteti HR, Noel S, Racusen L, et al. The Nrf2 triterpenoid activator, CDDO-imidazolide, protects kidneys from ischemia-reperfusion injury in mice. Kidney Int. 2014;85:134-41.

3. Kensler TW, Wakabayashi N, Biswal S. Cell survival responses to environmental stresses via the Keap1-Nrf2-ARE pathway. Annu Rev Pharmacol Toxicol. 2007:47:89-116.

4. Itoh K, Wakabayashi N, Katoh Y, Ishii T, Igarashi K, Engel JD, Yamamoto M. Keap1 represses nuclear activation of antioxidant responsive elements by Nrf2 through binding to the amino-terminal Neh2 domain. Genes Dev. 1999;13:76-86.

5. Baird L, Dinkova-Kostova AT. The cytoprotective role of the Keap1-Nrf2 pathway. Arch Toxicol. 2011;85:241-72.

6. Shelton LM, Park BK, Copple IM. Role of Nrf2 in protection against acute kidney injury. Kidney Int. 2013;84:1090-5.

7. Tanaka Y, Maher JM, Chen C, Klaassen CD. Hepatic ischemia-reperfusion induces renal heme oxygenase-1 via NF-E2-related factor 2 in rats and mice. Mol Pharmacol. 2007;71:817-25.

8. Li M, Liu RM, Timblin CR, Meyer SG, Mossman BT, Fukagawa NK. Age affects ERK1/2 and Nrf2 signaling in the regulation of GCLC expression. J Cell Physiol. 2006;206:518-25.

9. Wakabayashi N, Itoh K, Wakabayashi J, Motohashi H, Noda S, Takahashi S, et al. Keap1-null mutation leads to postnatal lethality due to constitutive Nrf2 activation. Nat Genet. 2003;35:238-45.

10. Kong $X$, Thimmulappa R, Craciun F, Harvey C, Singh A, Kombairaju P, et al. Enhancing Nrf2 pathway by disruption of Keap1 in myeloid leukocytes protects against sepsis. Am J Respir Crit Care Med. 2011;184:928-38.

11. Noel S, Martina MN, Bandapalle S, Racusen LC, Potteti HR, Hamad ARA, et al. T lymphocyte-specific activation of Nrf2 protects from AKI. J Am Soc Nephrol. 2015;26:2989-3000.

12. Blake DJ, Singh A, Kombairaju P, Malhotra D, Mariani TJ, Tuder RM, et al. Deletion of Keap1 in the lung attenuates acute cigarette smoke-induced oxidative stress and inflammation. Am J Respir Cell Mol Biol. 2010;42:524-36. 
13. Shao X, Somlo S, Igarashi P. Epithelial-specific Cre/lox recombination in the developing kidney and genitourinary tract. J Am Soc Nephrol. 2002;13:1837-46.

14. Zhang Z, Pascuet E, Hueber PA, Chu L, Bichet DG, Lee TC, et al. Targeted inactivation of EGF receptor inhibits renal collecting duct development and function. J Am Soc Nephrol. 2010;21:573-8.

15. Fujita H, Hamazaki Y, Noda Y, Oshima M, Minato N. Claudin-4 deficiency results in urothelial hyperplasia and lethal hydronephrosis. PLoS One. 2012;7(12):e52272

16. lizuka-Kogo A, Akiyama T, Senda T. Decreased apoptosis and persistence of the common nephric duct during the development of an aberrant vesicoureteral junction in Dlg1 genetargeted mice. Anat Rec (Hoboken). 2013;296:1936-42.

17. Chen CP, Chang SD, Wang TH, Wang LK, Tsai JD, Liu YP, et al. Detection of recurrent transmission of 17q12 microdeletion by array comparative genomic hybridization in a fetus with prenatally diagnosed hydronephrosis, hydroureter and multicystic kidney, and variable clinical spectrum in the family. Taiwan J Obstet Gynecol. 2013:52:551-7.

18. Stark S, Winkelmann B, Kluthe C, Roigas J, Querfeld U, Müller D. Polycythemia and increased erythropoietin in a patient with chronic kidney disease. Nat Clin Pract Nephrol. 2007:3:222-6.

19. Toyama K, Mitus WJ. Experimental renal erythrocytosis. 3. Relationship between the degree of hydronephrotic pressure and the production of erythrocytosis. J Lab Clin Med. 1966;68:740-52.

20. Fisher JW, Schofield R, Porteous DD. Effects of renal hypoxia on erythropoietin production. Br J Haematol. 1965;11:382-8.

21. Eckardt KU, Kurtz A. Regulation of erythropoietin production. Eur J Clin Invest 2005;35 Suppl 3:13-9.

22. Maxwell PH, Wiesener MS, Chang GW, Clifford SC, Vaux EC, Cockman ME, et al. The tumour suppressor protein VHL targets hypoxia-inducible factors for oxygen-dependentproteolysis. Nature. 1999;399:271-5.

\section{Submit your next manuscript to BioMed Central and we will help you at every step:}

- We accept pre-submission inquiries

- Our selector tool helps you to find the most relevant journal

- We provide round the clock customer support

- Convenient online submission

- Thorough peer review

- Inclusion in PubMed and all major indexing services

- Maximum visibility for your research

Submit your manuscript at www.biomedcentral.com/submit

) Biomed Central 